\title{
La decisión de estudiar medicina:
}

\section{características}

\section{The decision to study Medicine: characteristics}

Marisol Soria, Manuel Guerra, Ignacio Giménez y Jesús Fernando Escanero

Departamento de Farmacología y Fisiología, Facultad de Medicina, Zaragoza.

En el presente trabajo se analizan las distintas características que definen la decisión de estudiar Medicina. A través de una encuesta respondida por los estudiantes de segundo curso de Medicina se ha evaluado el momento de la decisión, los factores que han influido en la misma, los motivos por los que se ha elegido y el patrón de selección. Se presenta un análisis descriptivo de las variables indicadas y de la relación existente entre ellas, así como de la posible influencia del sexo en cada una. Entre los resultados obtenidos se observa que un gran porcentaje de estudiantes toman la decisión de estudiar Medicina antes que el resto de población preuniversitaria opta por la carrera a estudiar. Entre los estudiantes de Medicina, los que se deciden antes presentan diferencias significativas respecto a los factores de influencia, los motivos de la decisión y el patrón de selección. Por otro lado, se encuentra que los principales motivos por los que se elige esta carrera son los de tipo altruista y humanitario. Las mujeres presentan diferencias respecto a la especialidad que les gustaría hacer y en ellas los motivos instrumentales son significativamente inferiores respecto a los varones.

En conclusión, los estudiantes de Medicina presentan un alto grado de vocación social, que se ha visto
Correspondencia:

Marisol Soria

c/ Domingo Miral s.n. Zaragoza 50009

Tfno:976-761699

email:msoria@unizar.es incluso reforzada por la masiva incorporación de la mujer a esta carrera.

Palabras clave: carrera de Medicina, estudiantes de Medicina, elección, factores de influencia, motivos, patrones de selección y sexo.

The present paper analyses the different factors that govern the decision to study Medicine. Second year medical students answered a questionnaire designed to analyze the moment when the decision was taken, the factors influencing this decision, their reasons for choosing Medicine and the pattern of selection. A descriptive analysis of the indicated variables and the relation between them was carried out, taking into account the possible influence of sex for all variables. The results obtained show that a high percentage of students that choose to study Medicine take their decision before the rest of the student population decides on what degree to study. Those students who decide earlier present significant differences in terms of the factors that influenced their decision and the pattern of selection. Moreover, it has been found that the principal reasons for choosing this degree are altruistic and humanitarian. Women show different results regarding the medical speciality that they would like to pursue and place significantly less importance on material factors than their male counterparts. In conclusion, medical students present a high degree of social vocation that has been reinforced by the substantial incorporation of women into this area of study.

Key words: Medical degree, Medical students, election, factors of influence, reasons, patterns of selection and sex. 


\section{INTRODUCCIÓN}

En las últimas décadas se está asistiendo a la entrada masiva de la población juvenil a la universidad lo que ha provocado la saturación de muchas carreras, entre ellas la de Medicina. El numerus clausus, como medida preventiva de este fenómeno, es un criterio de selección basado en notas académicas previas, lo que supone que las personas que acceden a esta carrera son las que tienen expedientes académicos más altos. Como viene siendo habitual en estos últimos años, los estudios de Medicina, junto con otras carreras relacionadas con la salud, han alcanzado las notas de corte más elevadas en las universidades españolas: así, en el curso 2002-3, Fisioterapia y Medicina fueron las dos únicas titulaciones que superaron los 7 puntos como nota exigida para matricularse en la Universidad de Zaragoza, siendo la Universidad de Las Palmas la que registró la nota de corte en Medicina más elevada de España: $8,31^{1}$. De esta forma, puede considerarse a priori, que los estudiantes seleccionados son los jóvenes más preparados y con gran talento para el estudio. Sin embargo, este criterio de selección no tiene en cuenta si las personas que acceden a esta carrera son realmente las de mayor vocación y poseen las dotes personales y sociales necesarias para el trato con el paciente ${ }^{2,3}$. Debe tenerse en cuenta que la vocación en Medicina es servicio y en este sentido la Medicina es una profesión de servicio a las personas y a la sociedad que requiere un cuidado y atención especial. Por lo tanto, además de contar con los jóvenes mejor preparados y de mayor talento para adquirir los conocimientos necesarios, estos deben tener o adquirir una especial capacidad para escuchar, comunicar y disponerse a servir a quien lo requiera ${ }^{2,3}$ Por otra parte, se está asistiendo a un fenómeno cada vez más acentuado de incorporación de la mujer a la carrera de Medicina, donde actualmente supera el $70 \%$ de los estudiantes ${ }^{4-7}$. Si se tiene en cuenta que el proceso vocacional, que va dirigido a la plena realización de todas las posibilidades del individuo, esta influido por el sexo ${ }^{8-10}$, puede pensarse que el perfil vocacional de las Facultades de Medicina está siendo influido por la variable sexo y que los hombres y mujeres ingresan en la carrera de Medicina por motivos distintos.

Si a esto se une que el rol tradicional del médico ha cambiado y que hoy día el reconocimiento social de la profesión también es distinto al de antaño, así como su poder adquisitivo, se puede pensar que los motivos por los que los estudiantes eligen cursar Medicina son presumiblemente diferentes a los de otras épocas y que la decisión de estudiar esta carrera viene influenciada por una serie de determinantes personales y condicionantes externos bien distintos a los que pudieran estar presentes en el pasado ${ }^{4,11,12}$

Teniendo en cuenta todo lo anterior, los objetivos del presente estudio consisten en averiguar en qué momento se toma la decisión de estudiar Medicina y qué factores han influido sobre la misma, establecer los patrones de selección e identificar los motivos por los que se eligió esta carrera. Finalmente, se intentará comprobar qué relaciones existen entre los parámetros estudiados y se analizarán las posibles influencias del perfil demográfico y especialmente de la variable sexo sobre esta decisión.

\section{MATERIAL Y MÉTODOS}

\section{Muestra}

La población estudiada esta constituida por los alumnos de Medicina de segundo curso de la Facultad de Medicina de Zaragoza.

No hubo una selección a priori de la muestra aplicando criterios de inclusión o exclusión puesto que interesaba analizar todas las variaciones posibles del conjunto de la población estudiantil. Para la inclusión en el estudio se tuvo en cuenta únicamente la asistencia a clase en un día determinado del curso escolar al finalizar el primer trimestre. De los 129 alumnos matriculados en segundo curso de medicina han participado $105(\mathrm{n}=105)$, lo que representa un $81 \%$.

\section{Instrumento utilizado y procedimiento}

Los datos se recogieron en una encuesta anónima que los estudiantes respondieron en presencia de un investigador del equipo encargado de responder a las consultas y controlar cualquier comunicación entre los alumnos.

Los estudiantes fueron informados del propósito del estudio y se pidió su participación voluntaria y anónima. Posteriormente se les entregó la encuesta y explicó el contenido de la misma.

La encuesta antes de suministrarse a la población muestral fue ensayada en un grupo de alumnos de internos pertenecientes al Departamento de Fisiología de la Facultad de Medicina de Zaragoza.

El cuestionario constaba de 32 preguntas, fue diseñado por nuestro equipo de trabajo y se dividió en dos apartados: el primero, sobre las características sociodemográficas $\mathrm{y}$, el segundo, sobre aspectos relacionados con la decisión de cursar estudios de 
Medicina. El apartado sociodemográfico consta de 9 preguntas y se elaboró a partir de encuestas anteriores aplicadas por el grupo de trabajo en estudios previos $^{5,6,7}$. Tiene por finalidad delimitar el perfil demográfico y social de los encuestados. El segundo apartado de la encuesta se definió teniendo en cuenta los estudios realizados por Feather ${ }^{13}$ y Ewan ${ }^{14} \mathrm{y}$ está dividido en cuatro bloques con la finalidad de analizar diversas variables referentes a la decisión de estudiar Medicina. El primer bloque tiene por objeto conocer las etapas en la decisión de estudiar Medicina y está constituido por siete preguntas. El segundo intenta averiguar los factores que han influido en esta decisión y consta de nueve cuestiones. El tercero, de cuatro preguntas, está destinado a definir los patrones de selección de la carrera. Y finalmente el último, con tres preguntas (una de ellas de opción abierta), explora los motivos de tal decisión, teniendo que valorar en una de ellas, entre 0 y 5,18 posibles razones por las que ha elegido Medicina.

Los datos obtenidos se han procesado con el paquete estadístico SPPS. Además del cálculo de medias, desviaciones estándar y porcentajes se han utilizado diferentes pruebas estadísticas según las características de las variables que se han estudiado y de las comparaciones establecidas. Las pruebas empleadas han sido: prueba t de Student, ANOVA, Coeficiente de Correlación de Pearson, y el estadístico de Chi-cuadrado. En todos los casos se ha trabajado con un nivel de significación del $95 \%$ $(\mathrm{p} \leq 0,05)$.

\section{RESULTADOS}

\section{I.- Perfil demográfico y social}

Entre las características socio demográficas de la muestra utilizada destaca el alto porcentaje de mujeres observado (75\%). Comparándolo con el de la población universitaria en general, que es de un $55 \%$, la diferencia resulta muy significativa $(\mathrm{p}<0,01)$. La edad media de los encuestados fue de 19 años.

Del reto de datos socidemográficos recogidos interesa únicamente mencionar que el $18 \%$ de los estudiantes tenían familiares médicos en primer grado.

II.- Características de la decisión de estudiar Medicina

A.- Etapas de la decisión de estudiar Medicina

En este apartado se preguntó a los estudiantes a que edad habían pensado por primera vez hacer la carrera de Medicina y a que edad se habían decidi-

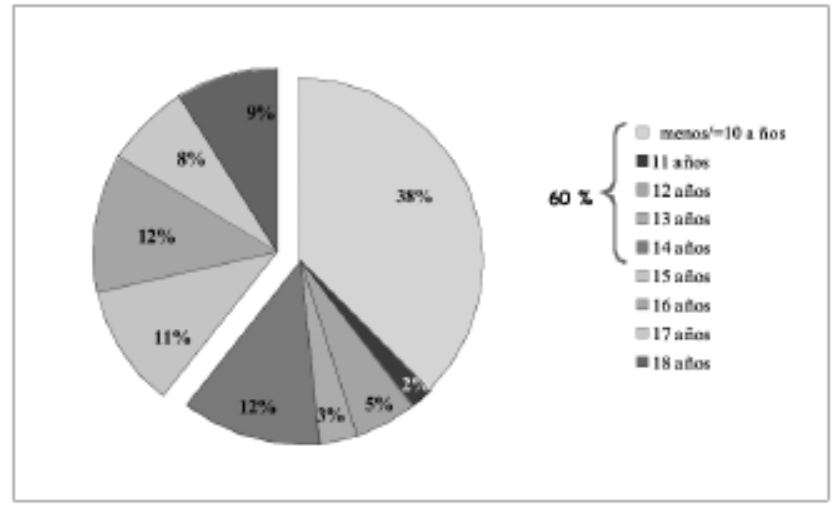

Figura 1. Edad a la que los estudiantes pensaron estudiar Medicina

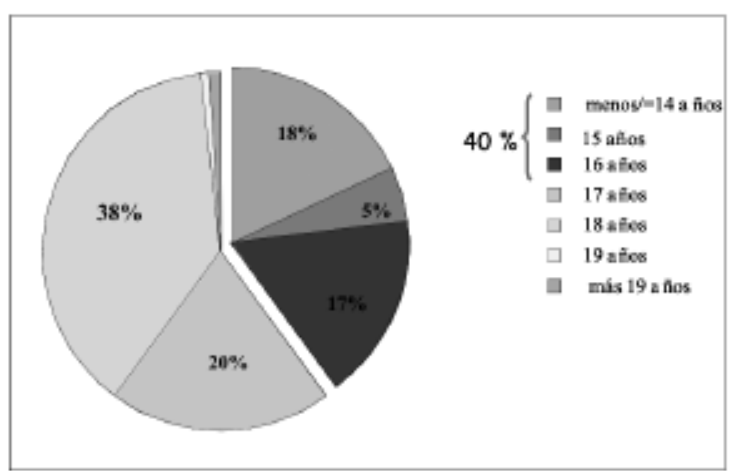

Figura 2. Edad a la que los estudiantes tomaron la decisión de estudiar Medicina

do definitivamente. Como puede observarse en la figura 1, más de la mitad de los estudiantes (60\%) pensaron estudiar Medicina por primera vez antes de los 14 años y un 38\% de ellos antes incluso de los 10 años. Respecto a la decisión definitiva (figura 2) un $40 \%$ de los encuestados la tomó antes de los 16 años y el $60 \%$ restante lo hizo a los 17 ó 18 años. Dichos datos resultan significativamente distintos respecto al resto de población preuniversitaria, que toma la decisión mayoritariamente a los 17 ó 18 años, es decir, el año antes de su ingreso en la universidad. De los estudiantes que pensaron precozmente estudiar medicina (a los 10 años o antes) el $78 \%$ también lo decidió pronto: antes de los 16 años, siendo esta correlación significativa $(\mathrm{p}<0,05)$.

La existencia de familiares médicos en el entorno no marcó diferencias significativas en cuanto a la edad de pensar o decidir estudiar la carrera.

Ante la pregunta de si sabía a que especialidad le gustaría dedicarse, el $55 \%$ de los estudiantes encuestados ya conocía la especialidad que le gustaría hacer, siendo los estudiantes que habían pensado y decidido precozmente los que en mayor proporción tenían decidida la especialidad. Estas diferen- 
cias son significativas respecto al grupo de estudiantes que piensa y/o decide tardíamente $(\mathrm{p}<0,05)$.

Finalmente, en cuanto a las preferencias relativas a especialidades ocupan el primer lugar las especialidades quirúrgicas, seguidas de cerca por la pediatría y la psiquiatría. Aquí las diferencias significativas fueron determinadas por el sexo en lo que se refiere a las especialidades elegidas en segundo y tercer lugar, ya que los varones se decantaron a favor de las especialidades médicas y de la traumatología.

\section{B.- Factores influyentes en la decisión}

El $70 \%$ de los encuestados señaló la existencia de algún factor influyente en su decisión de estudiar Medicina, mientras que el $30 \%$ restante declaró no haber sido influido por ninguno y haber llegado a la decisión por si mismos.

Como se observa en la figura 3 , dentro de los factores de influencia destaca en primer lugar la presión familiar, que fue señalada por el $16 \%$. Le siguió el contacto con la enfermedad $(15 \%)$ y la existencia de familiares médicos en el entorno (12\%). Un $11 \%$ de los estudiantes reconoció influencias de la televisión y un $10 \%$ y $5 \%$ de ellos dijo haber sido influido por tutores y amigos, respectivamente.

Se puso especial interés en valorar la posible influencia de la existencia de familiares médicos en el entorno del estudiante. El $18 \%$ de los estudiantes tenían familiares médicos en primer grado (padres, abuelos, hermanos). Se observaron diferencias significativas entre los estudiantes que tienen familiares médicos en primer grado y los que no tienen $(\mathrm{p}<0,05)$. Cuando existían este era el principal factor influyente $(90 \%)$, y cuando no predominó el haber llegado a la decisión por ellos mismos seguido del contacto con la enfermedad y la presión familiar.

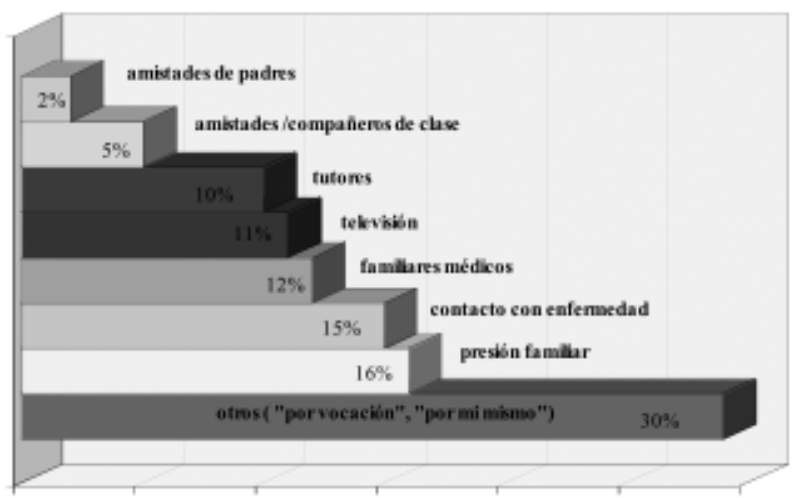

Figura 3. Factores que han influido en la decisión de estudiar Medicina
Respecto a la existencia o no de personas que hubieran influido en su decisión, el $45 \%$ dijo no haberlas tenido, mientras el $55 \%$ restante reconoció influencias personales, siendo la madre la que aparece en el $35 \%$ de las respuestas. En esta pregunta, la existencia de familiares médicos también supuso diferencias significativas $(\mathrm{p}<0,05)$ ya que este grupo presentó influencias personales mas marcadas $(65 \%)$.

La edad de decisión determinó la existencia de diferencias significativas en los factores de influencia $(p<0,05)$. Los que decidieron antes estaban más influenciados por el contacto con la enfermedad y la televisión, mientras que los que decidieron tardíamente lo hicieron más influenciados por la presión familiar y los tutores.

\section{C.- Patrones de selección}

Preguntando a los estudiantes que participaron en la encuesta por la preferencia de la selección de la carrera de Medicina, el 50\% de los encuestados consideraron que esta era la única carrera que podrían haber elegido, el $44 \%$ manifestaron que Medicina era una entre varias opciones y el 6\% señaló que no tenía claro que Medicina pudiera satisfacerles plenamente.

En la pregunta sobre la existencia en algún momento de dudas en la elección, el $58 \%$ declaró no haberlas tenido en ningún momento.

En la cuestión en la que se les planteó qué harían si se vieran obligados a tener que abandonar temporalmente los estudios de Medicina, la gran mayoría (87\%) contestó que retomaría los estudios de Medicina una vez solucionado el problema, mientras que el $10 \%$ haría otra carrera mas corta y solamente el 3\% dijo que abandonaría los estudios.

Se comprobó una correlación significativa $(\mathrm{p}<0,05)$ entre las distintas respuestas a las tres preguntas anteriores. Así, el grupo que consideraba la Medicina como única carrera no había tenido dudas de su elección y todos ellos retomarían los estudios de Medicina. El grupo que pensaba que Medicina era una entre varias opciones presentaba mayor porcentaje de dudas y, aunque la mayoría retomaría la carrera, aparece un pequeño porcentaje que indicó que haría una carrera más corta. Por el contrario, el grupo que no tenía claro que la Medicina pudiera satisfacerle es en el que se observó mayor proporción de dudas y el único que respondió que abandonarían la carrera.

Se encontraron también diferencias significativas respecto a la edad de pensar y de decidir estudiar 
Tabla 1. Media y ranking de la valoración de los 18 motivos surgidos por los que se ha elegido la carrera de Medicina y diferencias por sexos (media).

\begin{tabular}{|c|c|c|c|c|c|}
\hline & \multirow{2}{*}{ media } & \multirow{2}{*}{ ranking } & \multicolumn{2}{|c|}{ media } & \multirow{2}{*}{$\mathrm{p}$} \\
\hline & & & hombres & mujeres & \\
\hline Ejercer dirección (liderazgo) & 1,34 & 16 & 1,59 & 1,07 & 0,04 \\
\hline Ser original y creativo & 1,74 & 15 & 2,06 & 1,48 & 0,01 \\
\hline Libre de supervisión & 1,76 & 13 & 2,00 & 1,50 & 0,03 \\
\hline Evitar trabajos de alta presión que te exigen demasiado & 1,05 & 17 & 1,06 & 0,94 & 0,55 \\
\hline Trabajar con personas & 3,63 & 2 & 3,15 & 3,58 & 0,02 \\
\hline Satisfacer a padres o familiares & 1,88 & 12 & 1,46 & 1,78 & 0,25 \\
\hline Adquisición de alto estatus social & 1,89 & 11 & 1,87 & 1,55 & 0,14 \\
\hline Seguridad económica & 2,65 & 9 & 2,50 & 2,36 & 0,40 \\
\hline Tener probabilidad de éxito & 2,31 & 10 & 2,28 & 2,08 & 0,29 \\
\hline Tener fama & 0,93 & 18 & 1,15 & 0,71 & 0,06 \\
\hline Ganar mucho dinero & 1,75 & 14 & 1,90 & 1,41 & 0,04 \\
\hline Ayudar a los demás o a la sociedad & 3,74 & 1 & 3,41 & 3,68 & 0,07 \\
\hline Expresar valores que pueden ser modélicos para la gente & 3,13 & 7 & 2,75 & 2,90 & 0,60 \\
\hline Contribuir a mejorar la sociedad & 3,42 & 3 & 3,12 & 3,33 & 0,37 \\
\hline Ser trabajo de gran estímulo intelectual & 3,16 & 6 & 3,09 & 2,94 & 0,26 \\
\hline Poder ejercitar habilidades, destrezas y capacidades personales & 3,33 & 4 & 3,03 & 3,15 & 0,57 \\
\hline Profesión de desafios o retos & 3,08 & 8 & 2,90 & 2,95 & 0,96 \\
\hline Deseo de progreso constante & 3,19 & 5 & 2,96 & 3,12 & 0,34 \\
\hline
\end{tabular}

Medicina. Las personas que pensaron y decidieron antes son las que en mayor porcentaje manifestaron que era la única carrera que podía satisfacerles. Un hecho similar se observó en el grupo que tenía familiares médicos.

La existencia de familiares médicos en el entorno más cercano supuso diferencias significativas con respecto a esta cuestión, ya que mayoritariamente respondieron que seria la única carrera que elegirían.

\section{D.- Motivos de elección}

Cuando se preguntó por el grupo de razones por las que habían elegido la carrera, el $68 \%$ señaló en primer lugar las definidas como altruistas (ayudar a la gente y a los demás), un $25 \%$ las definidas como intelectuales (profesión de desafíos, estimulante, científica y exigente) y el tercer y cuarto lugar lo ocuparon las razones personales (presión familiar, contacto con enfermedad, familiares médicos) e instrumentales (prestigio, estatus, nivel económico, buenas salidas profesionales) con un $4 \%$ cada una de ellas. Estos datos concuerdan con los obtenidos en la siguiente pregunta donde de forma abierta se indagó por la razón principal por la que habían elegido la carrera, siendo la respuesta mas señalada ayudar a los demás, seguida de contribuir a mejorar la sociedad y trabajar con personas.

La tabla 1 presenta la valoración media de cada una de 18 razones posibles sugeridas para haber elegido Medicina. Se observa que los motivos altruistas y sociales son los que aparecieron en los primeros lugares con un mayor porcentaje. Ejercer el liderazgo, trabajar sin presión y alcanzar la fama fueron las razones menos valoradas.

De nuevo se encontraron diferencias entre ambos sexos. Los estudiantes de sexo masculino concedieron mayor importancia a motivos instrumentales mientras que los estudiantes de sexo femenino tuvieron preferencia por motivos altruistas y sociales. No obstante, estas diferencias en ítems específicos no deben hacernos perder de vista el contexto general que ponía de manifiesto que las razones altruistas y de tipo social eran las principales en ambos sexos.

\section{DISCUSIÓN}

El estudio descriptivo de estudiantes de la Facultad de Medicina de Zaragoza en los primeros cursos de carrera permite comprobar el elevado porcentaje de mujeres que cursa la misma, como ya había sido puesto de manifiesto en estudios anterio$\operatorname{res}^{4,5,6,7}$. Este porcentaje es mucho mayor que en otros estudios universitarios (75\% vs $55 \%$ ), siendo esta diferencia significativa ${ }^{1}$

Este hecho podría estar causado por la circunstancia de la incorporación de la mujer al mundo laboral y universitario que como es lógico también afecta a la esfera de la Medicina. Además, el sexo es una variable que influye en la personalidad del sujeto, en las expectativas de futuro y en su conducta vocacional ${ }^{8,9,10}$. En este sentido la causa del gran 
porcentaje de mujeres quizá pueda encontrarse en la mayor tendencia del sexo femenino hacia los intereses altruistas y sociales como se ha verificado en este estudio. Es decir, si se tiene en cuenta que en Medicina el sexo femenino es mayoritario y se conoce la influencia del sexo en la vocación, se puede pensar que los distintos sexos se acercan a los estudios de Medicina desde supuestos diferentes y con motivaciones distintas como se ha puesto de manifiesto por los resultados que constatan una mayor tendencia del sexo masculino hacia los motivos de tipo instrumental e intelectual. Esta interpretación viene reportada por los trabajos de Feather ${ }^{13}$, Romer ${ }^{15}$ y Jordan et $\mathrm{al}^{16}$.

Por otra parte, el cambio de rol del medico actual, con una marcada tendencia al funcionariado que ha supuesto una estabilización en la escala social y adquisitiva, encaja mejor con las características de la personalidad femenina, claramente tendente a la búsqueda de la estabilidad y menos influida por motivos instrumentales ${ }^{10}$.

Este estudio también ha servido para poner de manifiesto las diferencias marcadas por el sexo en lo que se refiere a las preferencias por las distintas especialidades médicas, diferencias que se han venido objetivando reiteradamente en distintos trabajos $^{10,13,14,17,18}$.

Con respecto a la decisión de estudiar Medicina y en concreto a las etapas de tal decisión, es de destacar el alto porcentaje de estudiantes que toma la decisión definitiva en una edad anterior a lo que suele hacerlo el resto de población universitaria, lo que coincide con los datos aportados por otros estu$\operatorname{dios}^{19}$. Estos estudiantes presentan diferencias respecto a los que toman la decisión en el último año de bachillerato. Dichas diferencias están en relación con los factores de influencia, los patrones de selección, la existencia de dudas en algún momento y el saber o no qué tipo de especialidad les gustaría hacer en el futuro. De esta manera los resultados corroboran, como parecería lógico suponer, que los estudiantes que deciden antes son los que piensan que la carrera de Medicina es la única que podían haber elegido, los que menos dudas han tenido hasta el momento y los que en mayor porcentaje han decidido hacer esta carrera por razones altruistas. Finalmente, los estudiantes que deciden antes saben mayoritariamente qué tipo de especialidad quieren hacer en el futuro. Todo esto sugiere un fuerte componente vocacional.

Los factores que influyen en la decisión de hacer una carrera están relacionados con las futuras moti- vaciones que respaldaran la decisión tomada, por ello en este estudio se abordaron los posibles factores influyentes en la decisión y posteriormente los motivos de la misma. Como señaló Maslow ${ }^{20}$, entender lo que motiva a la conducta humana es complicado, la fuerza motriz que motiva a las personas deriva de una jerarquía de necesidades y la motivación es algo que afecta a todos los aspectos de nuestra vida incluida la elección de la carrera. Para Blustein $^{21}$, en la elección de una carrera intervienen dos tipos de motivaciones la intrínseca y la extrínseca. La motivación intrínseca vendría definida por el hecho de realizar una actividad por el placer y satisfacción que uno experimenta mientras aprende, explora o trata de entender algo nuevo. De este modo puede definirse como el hecho de enrolarse en una actividad por el placer y la satisfacción experimentada cuando uno intenta realizar o crear algo. La motivación extrínseca abarca un amplia gama de conductas las cuales son medios para obtener un fin y no el fin en sí mismas. De todo esto puede concluirse que lo que motiva a una persona para elegir una carrera determinada está influenciado por multitud de factores.

En este sentido, las personas que dicen no haber tenido factores influyentes en su decisión y haber llegado a ésta por si mismos (30\% de la población muestral), podrían englobarse en el grupo en el que la motivación es mas bien de carácter intrínseco, motivados por la propia carrera; mientras que las que reconocen estar influenciadas por distintos factores podrían asociarse a motivación de origen extrínseco o intrínseco. Resulta llamativo el hecho de que los estudiantes con familiares médicos de primer grado toman la decisión por ellos mismos en un porcentaje significativamente inferior; probablemente también se trate de estudiantes con una fuerte motivación intrínseca lo que ocurre que la influencia del ambiente es muy marcada.

Respecto a las distintas motivaciones e intereses de los estudiantes de Medicina en los primeros años de carrera puede aseverarse que todos ellos están fuertemente motivados por un alto grado de razones altruistas y humanitarias, lo que permite afirmar que a pesar del sistema de acceso a los estudios de Medicina, las personas que hoy día están cursando esta carrera presentan una elevada motivación social, intelectual y científica, lo que está de acuerdo con los hallazgos obtenidos por Feather ${ }^{10}$. Este hecho desde nuestra consideración debe hacer reflexionar profundamente al profesorado en relación con su trabajo diario y su futura planificación curricular ya que 
la sociedad está poniendo en sus aulas a una gran parte de la juventud más preparada y motivada.

En conclusión, los resultados de este trabajo indican que en los estudiantes de Medicina de hoy día prima la dedicación a los demás y el compromiso con las personas, siendo la vocación social su fuerza motriz. El hecho de que la mujer este ocupando un alto porcentaje en la profesión ha supuesto que el componente vocacional se vea vigorosamente reforzado.

\section{BIBLIOGRAFÍA}

1. Estadística Universitaria: curso 2002-2003. Zaragoza: Gobierno de Aragón y Universidad de Zaragoza, 2002.

2. Mitchell KR, Hayes M. Student characteristics on entry to an innovative medical programme. Med Educ 1983; 17(2): 79-82.

3. O'Connell M, Beigton F. Student's career plans and the medical profession. J Med Educ 1979; 54: 509-11.

4. Ewan CE, Bennett MJ. Medicine in prospect- the first year student's view. Med Educ 1981; 15(5): 287-93.

5. Escanero J, Puzo J, Alda O, Soteras F. Estilos de aprendizaje de los estudiantes de la facultad de Medicina. Ed.: Instituto de Ciencias de la Educación. Universidad de Zaragoza. Zaragoza, 1987.

6. Alda O, Escanero J. Intereses y actitudes de los estudiantes de medicina del Colegio Universitario de Huesca por las asignaturas de primer ciclo. Ed.: Instituto de Ciencias de la Educación. Universidad de Zaragoza. Zaragoza, 1985.

7. Escanero J, Guerra M, Borque. L. Estilos de aprendizaje y formación de especialistas médicos. Ed.: Instituto de Ciencias de la Educación. Universidad de Zaragoza. Zaragoza, 1992.

8. Rivas E. Psicología vocacional: enfoques de asesoramiento. Madrid: ediciones Morata, 1993.
9. Castaño C. Psicologia y orientación vocacional, un enfoque interactivo. Madrid: ediciones Morova, 1985.

10. Mauleon-Moscardo PJ. Influencias del sexo en las preferencias vocacionales y rasgos de personalidad en los estudiantes de medicina. Actas Esp Psiquiatr 2003; 31 (1): 24-30.

11. Henderson MC, Hunt DK, Williams JW. General internists influence students to choose primary care careers: the power of role modelling. Am J Med 1996; 101: 648-653.

12. Kiessling C, Schubert B, Scheffner D, Burger W. Schooling, living conditions and study motivation of students partaking in the traditional or reformed course of instruction at the Charite Hospital. Dtsch Med Wochenschr 2003; 128(4): 135-40.

13. Feather NT. Values and atitudes of medical students. J Med Educ 1981; 56(10): 818-30.

14. Ewan C. Attitudes to social issues in medicine: a comparison of first-year medical students with first-year students in nonmedical faculties. Med Educ 1987; 21(1): 25-31.

15. Romer S. Motivations of woman over 30 for going to medical school. J Med Educ 1981; 56: 856-58.

16. Jordan J, Brown JB, Russell G. Choising family medicine. What influences medical students? Can Fam Physician 2003; 49: 1065-69.

17. Wendel T, Godellas C, Prinz R. Are there gender differences in choosing a surgical career? Surgery 2003; 134(4): 591-96.

18. Feng L, Ruzal-Shapiro C. Factor that influence radiologists' career choices. Acad Radiol 2003; 10(1): 45-51.

19. García JC. La decisión de estudiar medicina. Educ Med Salud 1970; 4(4): 277-94.

20. Maslow AH. Motivation and personality (2nd ed). New York: Harper and Row, 1970.

21. Blustein, DL. The relationship between motivational processes and career exploration. Journal of Vocational Behavior 1988; 32(3): 345-357. 